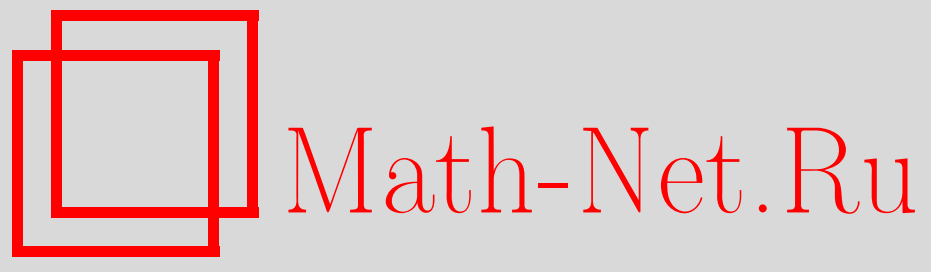

Ю. Л. Сачков, Симметрии и страты Максвелла в задаче об оптимальном качении сферы по плоскости, Матем. сб., 2010, том 201, номер 7, 99-120

DOI: https://doi.org/10.4213/sm7617

Использование Общероссийского математического портала Math-Net.Ru подразумевает, что вы прочитали и согласны с пользовательским соглашением http://www.mathnet.ru/rus/agreement

Параметры загрузки:

IP : 54.162 .127 .20

26 апреля 2023 г., 17:44:26

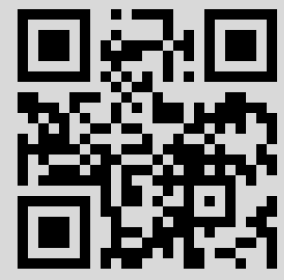




\section{Ю. Л. Сачков \\ Симметрии и страты Максвелла в задаче об оптимальном качении сферы по плоскости}

Рассматривается задача о качении сферы по плоскости без прокручивания и проскальзывания. Требуется перекатить сферу из одной контактной конфигурации в другую так, чтобы длина кривой, пробегаемой точкой контакта, была наименьшей. Экстремальные траектории в этой задаче были описаны А. Артуром, Дж. Уолшем и В. Джарджевичем.

В работе построены дискретные и непрерывные симметрии задачи, исследованы неподвижные точки их действия в прообразе и образе экспоненциального отображения. На основе этого анализа получены необходимые условия оптимальности - верхние оценки времени разреза вдоль экстремальных траекторий.

Библиография: 21 название.

Ключевые слова: оптимальное управление, геометрические методы, симметрии, качение поверхностей, эластики Эйлера.

\section{§1. Введение}

Рассматривается механическая система, состоящая из двух горизонтальных плоскостей и сферы, касающейся этих плоскостей. Нижняя плоскость неподвижна, а сфера катится без прокручивания и проскальзывания благодаря горизонтальному движению верхней плоскости. Состояние такой системы описывается точкой контакта сферы с нижней плоскостью и ориентацией сферы в трехмерном пространстве. Требуется перекатить сферу из заданного начального состояния в заданное терминальное состояние так, чтобы кривая, пробегаемая точкой контакта на плоскости, имела минимальную длину. Управлением является скорость верхней плоскости, или, что эквивалентно, скорость центра сферы.

Будет исследоваться кинематика данной системы, поэтому наличие верхней плоскости можно игнорировать и изучать качение сферы по (нижней) плоскости без прокручивания и проскальзывания. Отсутствие проскальзывания означает, что мгновенная скорость точки контакта сферы и плоскости равна нулю, а отсутствие прокручивания означает, что вектор угловой скорости сферы горизонтален. Качение одной поверхности по другой без прокручивания и проскальзывания моделирует работу руки робота-манипулятора. Задачи о таком движении вызывают большой интерес в механике, робототехнике и теории управления (см., например, работы [1]-[4]).

Работа выполнена при поддержке Российского фонда фундаментальных исследований (грант № 09-01-00246-a), а также Программы Президиума РАН "Математическая теория управления".

(C) Ю. Л. САчков, 2010 
Задача об оптимальном качении сферы по плоскости была поставлена в работе Дж. Хаммерсли [5]. А. Артур и Дж. Уолш (см. [6]) показали, что уравнения для экстремальных траекторий в этой задаче интегрируются в квадратурах, а В. Джарджевич (см. [7], [8]) провел качественное исследование этих траекторий и показал, что при оптимальном качении точка контакта сферы и плоскости движется по эластикам Эйлера (стационарным конфигурациям упругого стержня на плоскости; см. [9], [10]).

Вопрос об оптимальности экстремальных траекторий оставался открытым. Малые дуги экстремальных траекторий оптимальны, однако большие дуги, вообще говоря, нет. Точка, в которой экстремальная траектория теряет оптимальность, называется точкой разреза (по терминологии римановой геометрии). В настоящей работе начато исследование точек разреза и соответствующего времени разреза в задаче об оптимальном качении сферы по плоскости. Доказаны верхние оценки времени разреза вдоль экстремальных траекторий, т.е. необходимые условия оптимальности. Эти оценки получены благодаря исследованию дискретных симметрий задачи и соответствующих точек Максвелла (точек пересечения экстремальных траекторий с одинаковыми значениями функционала и времени). Этот подход был успешно применен к анализу нескольких задач оптимального управления (см. [11]-[13]); имеющийся опыт дает основания предполагать, что полученные верхние оценки времени разреза в действительности совпадают с временем разреза.

Перейдем к математической постановке задачи. Пусть $e_{1}, e_{2}, e_{3}$ - неподвижный правый репер в пространстве $\mathbb{R}^{3}$ такой, что векторы $e_{1}, e_{2}$ лежат в плоскости $\mathbb{R}^{2} \subset \mathbb{R}^{3}$, по которой катится сфера $S^{2}$ единичного радиуса, а вектор $e_{3}$ направлен в полупространство, содержащее эту сферу. Репер $e_{1}, e_{2}, e_{3}$ закреплен в точке $O \in \mathbb{R}^{2}$. Пусть $f_{1}, f_{2}, f_{3}$ - подвижный правый репер, закрепленный в катящейся сфере $S^{2}$. Обозначим координаты точки в $\mathbb{R}^{3}$ в базисе $e_{1}$, $e_{2}, e_{3}$ как $(x, y, z)$, а координаты этой точки в базисе $f_{1}, f_{2}, f_{3}$, перенесенном в точку $O$, как $(X, Y, Z)$. Таким образом,

$$
x e_{1}+y e_{2}+z e_{3}=X f_{1}+Y f_{2}+Z f_{3} .
$$

Пусть вращение трехмерного пространства, переводящее подвижный репер $f_{1}$, $f_{2}, f_{3}$ в неподвижный репер $e_{1}, e_{2}, e_{3}$, задается матрицей $R \in \mathrm{SO}(3)$ в подвижном репере:

$$
\left(\begin{array}{l}
X \\
Y \\
Z
\end{array}\right)=R\left(\begin{array}{l}
x \\
y \\
z
\end{array}\right) .
$$

Состояние системы "сфера $S^{2}$ и плоскость $\mathbb{R}^{2}$ " задается координатами $(x, y)$ точки контакта $S^{2}$ и $\mathbb{R}^{2}$ и матрицей вращения $R$. В качестве управлений будем использовать вектор $\left(u_{1}, u_{2}\right)$ скорости центра сферы. Как показано в работе [7], задача об оптимальном качении сферы по плоскости формализуется как следующая задача оптимального управления:

$$
\begin{gathered}
\dot{x}=u_{1}, \\
\dot{y}=u_{2}, \\
\dot{R}=R\left(u_{2} A_{1}-u_{1} A_{2}\right),
\end{gathered}
$$




$$
\begin{gathered}
Q=(x, y, R) \in M=\mathbb{R}^{2} \times \mathrm{SO}(3), \\
u=\left(u_{1}, u_{2}\right) \in \mathbb{R}^{2}, \\
Q(0)=Q_{0}=(0,0, \mathrm{Id}), \quad Q\left(t_{1}\right)=Q_{1}, \\
l=\int_{0}^{t_{1}} \sqrt{u_{1}^{2}+u_{2}^{2}} d t \rightarrow \min .
\end{gathered}
$$

Здесь и далее мы используем базисные матрицы в алгебре Ли so(3)

$$
A_{1}=\left(\begin{array}{ccc}
0 & 0 & 0 \\
0 & 0 & -1 \\
0 & 1 & 0
\end{array}\right), \quad A_{2}=\left(\begin{array}{ccc}
0 & 0 & 1 \\
0 & 0 & 0 \\
-1 & 0 & 0
\end{array}\right), \quad A_{3}=\left(\begin{array}{ccc}
0 & -1 & 0 \\
1 & 0 & 0 \\
0 & 0 & 0
\end{array}\right) .
$$

Допустимые управления измеримые существенно ограниченные, а допустимые траектории липшицевы.

Задача (1.1)-(1.7) есть левоинвариантная субриманова задача на группе Ли $M=\mathbb{R}^{2} \times \mathrm{SO}(3)$. Введем следующий левоинвариантный репер на этой группе Ли:

$$
e_{1}=\frac{\partial}{\partial x}, \quad e_{2}=\frac{\partial}{\partial y}, \quad V_{i}(R)=R A_{i}, \quad i=1,2,3 .
$$

В терминах левоинвариантных полей

$$
X_{1}=e_{1}-V_{2}, \quad X_{2}=e_{2}+V_{1}
$$

управляемая система (1.1)-(1.4) принимает вид

$$
\dot{Q}=u_{1} X_{1}(Q)+u_{2} X_{2}(Q), \quad Q \in M=\mathbb{R}^{2} \times \operatorname{SO}(3), \quad\left(u_{1}, u_{2}\right) \in \mathbb{R}^{2} .
$$

Функционал (1.7) есть функционал субримановой длины для левоинвариантной субримановой структуры, заданной полями $X_{1}, X_{2}$ как ортонормированным базисом:

$$
\begin{gathered}
l=\int_{0}^{t_{1}}\langle\dot{Q}, \dot{Q}\rangle^{1 / 2} d t \rightarrow \min , \\
\left\langle X_{i}, X_{j}\right\rangle=\delta_{i j}, \quad i, j=1,2 .
\end{gathered}
$$

Коммутаторы $\left[A_{i}, A_{j}\right]=A_{i} A_{j}-A_{j} A_{i}$ вычисляются следующим образом:

$$
\left[A_{1}, A_{2}\right]=A_{3}, \quad\left[A_{2}, A_{3}\right]=A_{1}, \quad\left[A_{3}, A_{1}\right]=A_{2} .
$$

Таблица умножения в алгебре Ли $L=\operatorname{span}\left(e_{1}, e_{2}, V_{1}, V_{2}, V_{3}\right)$ группы Ли $M$ имеет вид

$$
\text { ad } e_{i}=0, \quad\left[V_{1}, V_{2}\right]=V_{3}, \quad\left[V_{2}, V_{3}\right]=V_{1}, \quad\left[V_{3}, V_{1}\right]=V_{2} .
$$

В силу равенств

$$
\left[X_{1}, X_{2}\right]=V_{3}, \quad\left[X_{1}, V_{3}\right]=-V_{1}, \quad\left[X_{2}, V_{3}\right]=-V_{2}
$$

векторные поля $X_{1}, X_{2}$ в правой части системы (1.9) порождают алгебру Ли $L$. По теореме Рашевского-Чжоу (см. [4]) система (1.9) вполне управляема, т.е. любые две точки $Q_{0}, Q_{1} \in M$ можно соединить траекторией системы. Из теоремы 
Филиппова (см. [4]) следует существование оптимальных управлений в задаче (1.1)-(1.7) для любых $Q_{0}, Q_{1} \in M$ в классе существенно ограниченных измеримых управлений.

Настоящая работа продолжает исследования инвариантных субримановых задач на группах Ли, активно ведущиеся в рамках геометрической теории управления (см. [14]-[16]). Она имеет следующую структуру. В §2 к задаче об оптимальном качении сферы по плоскости применяется принцип максимума Понтрягина и выводятся дифференциальные уравнения для экстремальных траекторий (в удобных для нас терминах повторяется часть результатов В. Джарджевича [7], [8]). В частности, показано, что подсистема гамильтоновой системы принципа максимума для сопряженных переменных в подходящих координатах сводится к уравнению математического маятника, а проекции экстремальных траекторий на плоскость $(x, y)$ суть эйлеровы эластики. Вводится экспоненциальное отображение, параметризующее экстремальные траектории. В $\S 3$ симметрии семейства эластик на плоскости (вращения и отражения) продолжаются сначала до симметрий семейства экстремалей, а затем - до симметрий экспоненциального отображения (т.е. до отображений в прообразе и образе экспоненциального отображения, с ним коммутирующих). Описывается соответствующая группа $G$ симметрий экспоненциального отображения. В $\S 4$ получены основные результаты работы - необходимые условия оптимальности экстремальных траекторий в виде верхних оценок времени разреза. Известно, что в аналитической задаче экстремальная траектория не может быть оптимальной после точки пересечения с другой экстремальной траекторией с теми же значениями времени и функционала (такие точки называются точками Максвелла). Мы исследуем неподвижные точки действия группы $G$ в прообразе и образе экспоненциального отображения. На этой основе описаны точки Максвелла, соответствующие группе симметрий $G$ - совпадающие конечные точки экстремальных траекторий, переставляемых элементами этой группы. В качестве следствия получены верхние оценки времени разреза. Эти результаты получены в терминах кватернионов единичной длины, параметризующих вращения трехмерного пространства; в 55 напоминаются основные факты, связанные с этой параметризацией.

\section{§ 2. Экстремальные траектории}

Из неравенства Коши-Буняковского следует, что задача минимизации субримановой длины (1.7) равносильна задаче минимизации действия

$$
J=\frac{1}{2} \int_{0}^{t_{1}}\left(u_{1}^{2}+u_{2}^{2}\right) d t \rightarrow \min
$$

при фиксированном терминальном времени $t_{1}$.

Для отыскания оптимальных траекторий в задаче (1.1)-(1.6), (2.1) применим принцип максимума Понтрягина [17] в инвариантной формулировке, для этого напомним некоторые факты гамильтонова формализма на кокасательном расслоении (см. [4]). Рассмотрим кокасательное расслоение $T^{*} M$ гладкого многообразия $M$ с канонической проекцией $\pi: T^{*} M \rightarrow M, \pi(\lambda)=q$ для ковектора $\lambda \in T_{q}^{*} M$. Тавтологическая 1-форма $s \in \Lambda^{1}\left(T^{*} M\right)$ на кокасательном 
расслоении определена следующим образом. Пусть $\lambda \in T^{*} M$ и $v \in T_{\lambda}\left(T^{*} M\right)$, тогда $\left\langle s_{\lambda}, v\right\rangle=\left\langle\lambda, \pi_{*} v\right\rangle$ (в координатах $s=p d q$ ). Каноническая симплектическая структура на кокасательном расслоении $\sigma \in \Lambda^{2}\left(T^{*} M\right)$ определяется как $\sigma=d s$ (в координатах $\sigma=d p \wedge d q$ ). Любому гамильтониану $h \in C^{\infty}\left(T^{*} M\right)$ можно сопоставить гамильтоново векторное поле на кокасательном расслоении $\vec{h} \in \operatorname{Vec}\left(T^{*} M\right)$ по правилу $\sigma_{\lambda}(\cdot, \vec{h})=d_{\lambda} h$.

Принцип максимума Понтрягина для задачи (1.1)-(1.6), (2.1) имеет следующую инвариантную формулировку (см. [4]). Введем линейные на слоях кокасательного расслоения $T^{*} M$ гамильтонианы, соответствующие базисным полям:

$$
h_{i}(\lambda)=\left\langle\lambda, e_{i}\right\rangle, \quad i=1,2, \quad H_{i}(\lambda)=\left\langle\lambda, V_{i}\right\rangle, \quad i=1,2,3,
$$

а также семейство гамильтонианов

$$
\begin{aligned}
h_{u, \nu}(\lambda) & =\left\langle\lambda, u_{1} X_{1}+u_{2} X_{2}\right\rangle+\frac{\nu}{2}\left(u_{1}^{2}+u_{2}^{2}\right) \\
& =u_{1}\left(h_{1}-H_{2}\right)+u_{2}\left(h_{2}+H_{1}\right)+\frac{\nu}{2}\left(u_{1}^{2}+u_{2}^{2}\right), \\
\lambda \in & T^{*} M, \quad u=\left(u_{1}, u_{2}\right) \in \mathbb{R}^{2}, \quad \nu \in \mathbb{R} .
\end{aligned}
$$

ТеОРема 1 (принцип максимума Понтрягина). Пусть $Q_{t} u u(t), t \in\left[0, t_{1}\right]$, являются оптимальной траекторией и соответствующим оптимальным управлением в задаче (1.1)-(1.6), (2.1). Тогда существует нетривиальная липшицева кривая

$$
\left(\nu, \lambda_{t}\right) \neq 0, \quad \nu \in \mathbb{R}, \quad \lambda_{t} \in T_{Q_{t}}^{*} M,
$$

для которой выполнены условия

$$
\begin{gathered}
\dot{\lambda}_{t}=\vec{h}_{u(t), \nu}\left(\lambda_{t}\right)=u_{1}(t)\left(\vec{h}_{1}\left(\lambda_{t}\right)-\vec{H}_{2}\left(\lambda_{t}\right)\right)+u_{2}(t)\left(\vec{h}_{2}\left(\lambda_{t}\right)+\vec{H}_{1}\left(\lambda_{t}\right)\right), \\
h_{u(t), \nu}\left(\lambda_{t}\right)=\max _{u \in \mathbb{R}^{2}} h_{u, \nu}\left(\lambda_{t}\right), \quad t \in\left[0, t_{1}\right], \\
\nu \leqslant 0 .
\end{gathered}
$$

Удовлетворяющая теореме 1 кривая $\lambda_{t} \in T^{*} M$ называется экстремалью, а ее проекция $Q_{t}=\pi\left(\lambda_{t}\right) \in M-$ экстремальной траекторией (или субримановой геодезической).

С использованием координат $\left(h_{1}, h_{2}, H_{1}, H_{2}, H_{3}\right)$ в слоях кокасательного расслоения $T^{*} M$ гамильтонова система (2.2) принимает вид

$$
\begin{gathered}
\dot{h}_{1}=\dot{h}_{2}=0, \\
\dot{H}_{1}=u_{1} H_{3}, \\
\dot{H}_{2}=u_{2} H_{3}, \\
\dot{H}_{3}=-u_{1} H_{1}-u_{2} H_{2}, \\
\dot{Q}=u_{1} X_{1}+u_{2} X_{2} .
\end{gathered}
$$

2.1. Анормальный случай. Рассмотрим сначала случай $\nu=0$. Из условия максимума (2.3) следует, что вдоль анормальных экстремалей

$$
h_{1}-H_{2} \equiv 0, \quad h_{2}+H_{1} \equiv 0 .
$$


Дифференцируя эти тождества, в силу гамильтоновой системы (2.4)-(2.8) получаем выражения для вертикальных координат

$$
H_{1}=\text { const }, \quad H_{2}=\text { const }, \quad H_{3}=0, \quad h_{1}=H_{2}, \quad h_{2}=-H_{1} \text {, }
$$

для экстремальных управлений

$$
u_{1}=k H_{2}=\mathrm{const}, \quad u_{2}=-k H_{1}=\mathrm{const}, \quad k=\mathrm{const} \neq 0
$$

и для экстремальных траекторий

$$
x_{t}=u_{1} t, \quad y_{t}=u_{2} t, \quad R_{t}=\exp \left(t\left(u_{2} A_{1}-u_{1} A_{2}\right)\right) .
$$

В анормальном случае сфера равномерно катится по прямой. Любая анормальная траектория оптимальна, т.е. не содержит точек разреза.

2.2. Нормальный случай. Теперь рассмотрим случай $\nu=-1$. Из условия максимума (2.3) получаем

$$
u_{1}=h_{1}-H_{2}, \quad u_{2}=h_{2}+H_{1} .
$$

Максимизированный гамильтониан имеет вид

$$
H=\frac{1}{2}\left(\left(h_{1}-H_{2}\right)^{2}+\left(h_{2}+H_{1}\right)^{2}\right),
$$

и соответствующая гамильтонова система есть

$$
\begin{gathered}
\dot{h}_{1}=\dot{h}_{2}=0, \\
\dot{H}_{1}=\left(h_{1}-H_{2}\right) H_{3}, \\
\dot{H}_{2}=\left(h_{2}+H_{1}\right) H_{3}, \\
\dot{H}_{3}=-h_{1} H_{1}-h_{2} H_{2}, \\
\dot{Q}=\left(h_{1}-H_{2}\right) X_{1}+\left(h_{2}+H_{1}\right) X_{2} .
\end{gathered}
$$

Как всегда в субримановых задачах, можно ограничиться геодезическими единичной скорости, т.е. экстремальными траекториями, вдоль которых

$$
H=\frac{1}{2}\left(\left(h_{1}-H_{2}\right)^{2}+\left(h_{2}+H_{1}\right)^{2}\right) \equiv \frac{1}{2} .
$$

При таком ограничении удобно перейти в сопряженном пространстве от координат $\left(h_{1}, h_{2}, H_{1}, H_{2}, H_{3}\right)$ к новым координатам $(r, \alpha, \theta, c)$ :

$$
\begin{array}{cl}
h_{1}=r \cos \alpha, \quad h_{2}=r \sin \alpha, \\
h_{1}-H_{2}=\cos (\theta+\alpha), \quad h_{2}+H_{1}=\sin (\theta+\alpha), \\
c=H_{3} .
\end{array}
$$

После этого гамильтонова система для нормальных экстремалей (2.9)-(2.13) принимает следующую форму, которую мы и будем исследовать в настоящей 
работе:

$$
\begin{gathered}
\dot{\theta}=c, \\
\dot{c}=-r \sin \theta, \\
\dot{\alpha}=\dot{r}=0, \\
\dot{x}=\cos (\theta+\alpha), \\
\dot{y}=\sin (\theta+\alpha), \\
\dot{R}=R \Omega, \quad \Omega=\sin (\theta+\alpha) A_{1}-\cos (\theta+\alpha) A_{2} .
\end{gathered}
$$

Переменные $\alpha$ и $\theta$ однозначно определяются переменными $h_{1}, h_{2}, H_{1}, H_{2}$ по соотношениям (2.14), (2.15) лишь в случае $r^{2}=h_{1}^{2}+h_{2}^{2} \neq 0$. В этом случае соответствие между траекториями систем (2.9)-(2.13) и (2.16)-(2.21) взаимно однозначно.

Если $r^{2}=h_{1}^{2}+h_{2}^{2}=0$, то параметр $\alpha$ не определяется соотношениями (2.14). В этом случае будем считать, что угол $\alpha$ принимает произвольное значение на окружности $S^{1}=\mathbb{R} /(2 \pi \mathbb{Z})$. Тогда каждой траектории системы $(2.9)-(2.13)$ соответствует бесконечный набор траекторий системы (2.16)-(2.21) с одной и той же функцией $\theta_{t}+\alpha$. Неоднозначность соответствия между траекториями систем (2.9)-(2.13) и (2.16)-(2.21) сводится к заменам вида $\widetilde{\theta}_{t}=\theta_{t}+\gamma, \widetilde{\alpha}=\alpha-\gamma$.

Семейство нормальных экстремалей $\lambda_{t}$ параметризуется цилиндром $C$, состоящим из начальных точек $\lambda=\left.\lambda_{t}\right|_{t=0}$ :

$$
\begin{aligned}
C & =\left\{\lambda \in T_{Q_{0}}^{*} M \mid H(\lambda)=\frac{1}{2}\right\} \\
& \cong\left\{\left(h_{1}, h_{2}, H_{1}, H_{2}, H_{3}\right) \in \mathbb{R}^{5} \mid\left(h_{1}-H_{2}\right)^{2}+\left(h_{2}+H_{1}\right)^{2}=1\right\} \\
& \cong\left\{(\theta, c, \alpha, r) \mid \theta \in S^{1}, c \in \mathbb{R}, \alpha \in S^{1}, r \geqslant 0\right\} .
\end{aligned}
$$

Каждой начальной точке $\lambda \in C$ соответствует экстремаль $\lambda_{t}=e^{t \vec{H}}(\lambda)$. Основным объектом дальнейшего исследования будет экспоненциальное отображение Exp, переводящее пару, состоящую из начальной точки $\lambda \in C$ и момента времени $t>0$, в конец соответствующей экстремальной траектории:

$$
\begin{gathered}
\operatorname{Exp}(\lambda, t)=\pi \circ e^{t \vec{H}}(\lambda)=Q_{t}, \\
\operatorname{Exp}: N \rightarrow M, \quad N=C \times \mathbb{R}_{+}=\{(\lambda, t) \mid \lambda \in C, t>0\} .
\end{gathered}
$$

В случае $r=0$ эластика $\left(x_{t}, y_{t}\right)$ есть прямая (при $H_{3}=c=0$ ) или окружность (при $H_{3}=c \neq 0$ ); будем называть такие эластики вырожденными.

В случае $r \neq 0$ эластика $\left(x_{t}, y_{t}\right)$ принадлежит одному из четырех классов в зависимости от полной энергии $E=c^{2} / 2-r \cos \theta$ маятника (2.16), (2.17):

1) инфлексионный при $E \in(-r, r)$;

2) неинфлексионный при $E \in(r,+\infty)$;

3) критический при $E=r, c \neq 0$;

4) прямая при $E=-r$ и при $E=r, c=0$.

Эластики классов 1)-3) будем называть невырожденными. 


\section{§ 3. Непрерывные и дискретные симметрии}

Пусть обратимое отображение $g$ действует в прообразе и образе экспоненциального отображения:

$$
g: N \rightarrow N, \quad g: M \rightarrow M .
$$

Имеются в виду два отображения, но удобнее обозначать их одной буквой; область действия отображения $(N$ или $M)$ будет ясна из контекста. Отображение $g$ называется симметрией экспоненииального отображения, если $g \circ \mathrm{Exp}=$ $\operatorname{Exp} \circ g$, т.е. коммутативна следующая диаграмма:

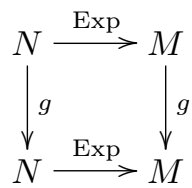

В этом параграфе построена группа симметрий экспоненциального отображения, соответствующая симметриям эластик (2.16)-(2.20) - вращениям и отражениям. В $\S 4$ на основе анализа действия этой группы будут получены оценки времени разреза в рассматриваемой задаче о качении сферы по плоскости.

3.1. Преобразование нормальных экстремалей. В этом пункте рассматриваются преобразования нормальных экстремалей $\lambda_{s}=\left(\theta_{s}, c_{s}, \alpha, r, Q_{s}\right)$, $s \in[0, t]$, т.е. траекторий гамильтоновой системы

$$
\begin{gathered}
\dot{\theta}_{s}=c_{s}, \\
\dot{c}_{s}=-r \sin \theta_{s}, \\
\dot{\alpha}=\dot{r}=0, \\
\dot{x}_{s}=\cos \left(\theta_{s}+\alpha\right), \\
\dot{y}_{s}=\sin \left(\theta_{s}+\alpha\right), \\
\Omega_{s}=\sin \left(\theta_{s}+\alpha\right) A_{1}-\cos \left(\theta_{s}+\alpha\right) A_{2}, \\
\dot{R}_{s}=R_{s} \Omega_{s}, \quad\left(x_{0}, y_{0}, R_{0}\right)=(0,0, \mathrm{Id}) .
\end{gathered}
$$

В вычислениях будет использоваться стандартный изоморфизм между алгебрами Ли so(3) и $\mathbb{R}^{3}$ (алгебраические операции - матричный коммутатор и векторное произведение соответственно); см., например, [4]. Матрице $A \in \operatorname{so}(3)$ сопоставляется вектор $\widetilde{A} \in \mathbb{R}^{3}$ по следующему правилу:

$$
A=\left(\begin{array}{ccc}
0 & -a_{3} & a_{2} \\
a_{3} & 0 & -a_{1} \\
-a_{2} & a_{1} & 0
\end{array}\right), \quad \widetilde{A}=\left(\begin{array}{l}
a_{1} \\
a_{2} \\
a_{3}
\end{array}\right) .
$$

Базисным матрицам $A_{i}$ соответствуют векторы $\widetilde{A}_{i}=e_{i}$ стандартного базиса в $\mathbb{R}^{3}$.

Следующие равенства будут полезны при вычислениях (см. [7]):

$$
\begin{gathered}
\widehat{e^{t B} A e^{-t B}}=e^{t B} \widetilde{A}, \quad A, B \in \operatorname{so}(3), \\
R R^{-1}=R \widetilde{A}, \quad R \in \mathrm{SO}(3), \quad A \in \operatorname{so}(3) .
\end{gathered}
$$


3.1.1. Вращения. Вращения эластик $\left(x_{s}, y_{s}\right)$ вокруг начала координат в плоскости $(x, y)$ порождают однопараметрическую группу симметрий траекторий гамильтоновой системы (3.1)-(3.7)

$$
\left\{\Phi^{\beta} \mid \beta \in S^{1}\right\}
$$

где вращение $\Phi^{\beta}$ определяется следующим образом:

$$
\begin{gathered}
\Phi^{\beta}:\left\{\lambda_{s} \mid s \in[0, t]\right\} \rightarrow\left\{\lambda_{s}^{\beta} \mid s \in[0, t]\right\}, \\
\lambda_{s}=\left(\theta_{s}, c_{s}, \alpha, r, Q_{s}\right), \quad Q_{s}=\left(x_{s}, y_{s}, R_{s}\right), \\
\lambda_{s}^{\beta}=\left(\theta_{s}^{\beta}, c_{s}^{\beta}, \alpha^{\beta}, r, Q_{s}^{\beta}\right), \quad Q_{s}^{\beta}=\left(x_{s}^{\beta}, y_{s}^{\beta}, R_{s}^{\beta}\right), \\
\theta_{s}^{\beta}=\theta_{s}, \quad c_{s}^{\beta}=c_{s}, \\
\alpha^{\beta}=\alpha+\beta, \\
\left(\begin{array}{l}
x_{s}^{\beta} \\
y_{s}^{\beta}
\end{array}\right)=\left(\begin{array}{cc}
\cos \beta & -\sin \beta \\
\sin \beta & \cos \beta
\end{array}\right)\left(\begin{array}{l}
x_{s} \\
y_{s}
\end{array}\right), \\
R_{s}^{\beta}=e^{\beta A_{3}} R_{s} e^{-\beta A_{3}}, \quad \Omega_{s}^{\beta}=e^{\beta A_{3}} \Omega_{s} e^{-\beta A_{3}} .
\end{gathered}
$$

ПреДЛОЖЕНИЕ 1. Если $\left\{\lambda_{s} \mid s \in[0, t]\right\}$ - траектория системы (3.1)-(3.7), то для любого $\beta \in S^{1}$ кривая $\left\{\lambda_{s}^{\beta} \mid s \in[0, t]\right\}$ есть также траектория этой системы.

ДокАЗАТЕЛЬСтво сводится к дифференцированию. Проверим, например, выполнение уравнений

$$
\dot{R}_{s}^{\beta}=R_{s}^{\beta} \Omega_{s}^{\beta}, \quad \Omega_{s}^{\beta}=\sin \left(\theta_{s}^{\beta}+\alpha^{\beta}\right) A_{1}-\cos \left(\theta_{s}^{\beta}+\alpha^{\beta}\right) A_{2} .
$$

Имеем

$$
\begin{aligned}
\Omega_{s}^{\beta} & =e^{\beta A_{3}}\left(\sin \left(\theta_{s}+\alpha\right) A_{1}-\cos \left(\theta_{s}+\alpha\right) A_{2}\right) e^{-\beta A_{3}} \\
& =\sin \left(\theta_{s}+\alpha\right) e^{\beta A_{3}} A_{1} e^{-\beta A_{3}}-\cos \left(\theta_{s}+\alpha\right) e^{\beta A_{3}} A_{2} e^{-\beta A_{3}}
\end{aligned}
$$

Из равенства (3.8) следует, что

$$
e^{\beta A_{3}} \widetilde{A_{1} e^{-\beta A_{3}}}=e^{\beta A_{3}} e_{1}=\cos \beta e_{1}+\sin \beta e_{2},
$$

поэтому $e^{\beta A_{3}} A_{1} e^{-\beta A_{3}}=\cos \beta A_{1}+\sin \beta A_{2}$. Аналогичными вычислениями получаем $e^{\beta A_{3}} A_{2} e^{-\beta A_{3}}=-\sin \beta A_{1}+\cos \beta A_{2}$. Следовательно,

$$
\begin{aligned}
\Omega_{s}^{\beta} & =\sin \left(\theta_{s}+\alpha\right)\left(\cos \beta A_{1}+\sin \beta A_{2}\right)-\cos \left(\theta_{s}+\alpha\right)\left(-\sin \beta A_{1}+\cos \beta A_{2}\right) \\
& =\sin \left(\theta_{s}+\alpha+\beta\right) A_{1}-\cos \left(\theta_{s}+\alpha+\beta\right) A_{2}=\sin \left(\theta_{s}^{\beta}+\alpha^{\beta}\right) A_{1}-\cos \left(\theta_{s}^{\beta}+\alpha^{\beta}\right) A_{2} .
\end{aligned}
$$

Далее,

$$
\dot{R}_{s}^{\beta}=e^{\beta A_{3}} \dot{R}_{s} e^{-\beta A_{3}}=e^{\beta A_{3}} R_{s} \Omega_{s} e^{-\beta A_{3}}=\left(e^{\beta A_{3}} R_{s} e^{-\beta A_{3}}\right)\left(e^{\beta A_{3}} \Omega_{s} e^{-\beta A_{3}}\right)=R_{s}^{\beta} \Omega_{s}^{\beta} .
$$

3.1.2. Отражения. В этом пункте отражения траекторий $\left(\theta_{s}, c_{s}\right)$ маятника (3.1), (3.2) в осях координат $\theta, c$ и в начале координат продолжаются до дискретных симметрий $\varepsilon^{1}, \varepsilon^{2}, \varepsilon^{3}$ семейства траекторий гамильтоновой системы (3.1)-(3.7):

$$
\begin{gathered}
\varepsilon^{i}:\left\{\lambda_{s} \mid s \in[0, t]\right\} \rightarrow\left\{\lambda_{s}^{i} \mid s \in[0, t]\right\}, \quad i=1,2,3, \\
\lambda_{s}=\left(\theta_{s}, c_{s}, \alpha, r, Q_{s}\right), \quad Q_{s}=\left(x_{s}, y_{s}, R_{s}\right), \\
\lambda_{s}^{i}=\left(\theta_{s}^{i}, c_{s}^{i}, \alpha^{i}, r, Q_{s}^{i}\right), \quad Q_{s}^{i}=\left(x_{s}^{i}, y_{s}^{i}, R_{s}^{i}\right) .
\end{gathered}
$$


Аналогичное продолжение для обобщенной задачи Дидоны (нильпотентной субримановой задачи с вектором роста $(2,3,5))$ было получено в работе [15].

Отражение $\varepsilon^{1}$. Отражению траекторий $\left(\theta_{s}, c_{s}\right)$ маятника $(3.1),(3.2)$ в оси координат $\theta$ соответствует следующая дискретная симметрия семейства экстремальных траекторий:

$$
\begin{gathered}
\theta_{s}^{1}=\theta_{t-s}, \quad c_{s}^{1}=-c_{t-s}, \quad \alpha^{1}=\alpha+\pi, \\
x_{s}^{1}=x_{t-s}-x_{t}, \quad y_{s}^{1}=y_{t-s}-y_{t}, \\
R_{s}^{1}=\left(R_{t}\right)^{-1} R_{t-s}, \quad \Omega_{s}^{1}=-\Omega_{t-s} .
\end{gathered}
$$

ПредлОЖЕНИЕ 2. Если $\left\{\lambda_{s} \mid s \in[0, t]\right\}$ - траектория системы (3.1)-(3.7), то кривая $\left\{\lambda_{s}^{1} \mid s \in[0, t]\right\}$ есть также траектория этой системы.

ДокАзАТЕЛьство. Проверим дифференциальные уравнения для отраженной кривой $\lambda_{s}^{1}$ :

$$
\begin{aligned}
\dot{\theta}_{s}^{1} & =-\dot{\theta}_{t-s}=-c_{t-s}=c_{s}^{1}, \\
\dot{c}_{s}^{1} & =c_{t-s}=-r \sin \theta_{t-s}=-r \sin \theta_{s}^{1}, \\
\dot{x}_{s}^{1} & =-\dot{x}_{t-s}=-\cos \left(\theta_{t-s}+\alpha\right)=\cos \left(\theta_{t-s}+\alpha+\pi\right)=\cos \left(\theta_{s}^{1}+\alpha^{1}\right), \\
\dot{y}_{s}^{1} & =-\dot{y}_{t-s}=-\sin \left(\theta_{t-s}+\alpha\right)=\sin \left(\theta_{t-s}+\alpha+\pi\right)=\sin \left(\theta_{s}^{1}+\alpha^{1}\right), \\
\Omega_{s}^{1} & =\sin \left(\theta_{s}^{1}+\alpha^{1}\right) A_{1}-\cos \left(\theta_{s}^{1}+\alpha^{1}\right) A_{2} \\
& =-\sin \left(\theta_{t-s}+\alpha\right) A_{1}+\cos \left(\theta_{t-s}+\alpha\right) A_{2}=-\Omega_{t-s}, \\
\dot{R}_{s}^{1} & =-\left(R_{t}\right)^{-1} \dot{R}_{t-s}=-\left(R_{t}\right)^{-1} R_{t-s} \Omega_{t-s}=R_{s}^{1} \Omega_{s}^{1} .
\end{aligned}
$$

Начальные условия для $Q_{s}^{1}$ также выполняются:

$$
\left.x_{s}^{1}\right|_{s=0}=x_{t}-x_{t}=0,\left.\quad y_{s}^{1}\right|_{s=0}=y_{t}-y_{t}=0,\left.\quad R_{s}^{1}\right|_{s=0}=\left(R_{t}\right)^{-1} \dot{R}_{t}=\mathrm{Id} .
$$

Для выяснения геометрического смысла действия симметрии $\varepsilon^{1}$ на эластики представим это действие следующим образом:

$$
\varepsilon^{1}:\left(\begin{array}{l}
x_{s} \\
y_{s}
\end{array}\right) \stackrel{(1)}{\longmapsto}\left(\begin{array}{l}
x_{t-s} \\
y_{t-s}
\end{array}\right) \stackrel{(2)}{\mapsto}\left(\begin{array}{c}
x_{t}-x_{t-s} \\
y_{t}-y_{t-s}
\end{array}\right) \stackrel{(3)}{\longmapsto}\left(\begin{array}{c}
x_{t-s}-x_{t} \\
y_{t-s}-y_{t}
\end{array}\right)=\left(\begin{array}{c}
x_{s}^{1} \\
y_{s}^{1}
\end{array}\right) .
$$

Здесь (1) есть обращение времени на эластике; $(2)$ - отражение плоскости $(x, y)$ в центре $p_{c}=\left(x_{t} / 2, y_{t} / 2\right)$ хорды эластики, т.е. отрезка, соединяющего ее начало $\left(x_{0}, y_{0}\right)=(0,0)$ и конец $\left(x_{t}, y_{t}\right) ;(3)$ - поворот эластики на угол $\pi$ вокруг начала координат $\left(x_{0}, y_{0}\right)=(0,0)$.

С другой стороны, отображение (3) ० (2) есть параллельный перенос начала эластики в начало координат.

Отражение $\varepsilon^{2}$. Отражение траекторий маятника $\left(\theta_{s}, c_{s}\right)$ в оси координат $c$ порождает следующую симметрию экстремальных траекторий:

$$
\begin{gathered}
\theta_{s}^{2}=-\theta_{t-s}, \quad c_{s}^{2}=c_{t-s}, \quad \alpha^{2}=\pi-\alpha \\
x_{s}^{2}=x_{t-s}-x_{t}, \quad y_{s}^{2}=y_{t}-y_{t-s}, \\
R_{s}^{2}=I_{2}\left(R_{t}\right)^{-1} R_{t-s} I_{2}, \quad \Omega_{s}^{2}=-I_{2} \Omega_{t-s} I_{2}, \\
I_{2}=I_{2}^{-1}=e^{\pi A_{2}}=\left(\begin{array}{ccc}
-1 & 0 & 0 \\
0 & 1 & 0 \\
0 & 0 & -1
\end{array}\right) .
\end{gathered}
$$


ПрЕДЛОЖЕНИЕ 3. Если $\left\{\lambda_{s} \mid s \in[0, t]\right\}$ - траектория системы (3.1)-(3.7), то кривая $\left\{\lambda_{s}^{2} \mid s \in[0, t]\right\}$ есть также траектория этой системы.

ДокАзАтЕльство. Аналогично доказательству предложения 2 проверим, например, уравнение для матрицы $R$ :

$$
\begin{gathered}
\Omega_{s}^{2}=\sin \left(\theta_{s}^{2}+\alpha^{2}\right) A_{1}-\cos \left(\theta_{s}^{2}+\alpha^{2}\right) A_{2}=\sin \left(\theta_{t-s}+\alpha\right) A_{1}+\cos \left(\theta_{t-s}+\alpha\right) A_{2}, \\
-I_{2} \Omega_{t-s} I_{2}=-e^{\pi A_{2}}\left[\sin \left(\theta_{t-s}+\alpha\right) A_{1}-\cos \left(\theta_{t-s}+\alpha\right) A_{2}\right] e^{-\pi A_{2}} \\
=-\sin \left(\theta_{t-s}+\alpha\right) e^{\pi A_{2}} A_{1} e^{-\pi A_{2}}+\cos \left(\theta_{t-s}+\alpha\right) e^{\pi A_{2}} A_{2} e^{-\pi A_{2}} \\
=\sin \left(\theta_{t-s}+\alpha\right) A_{1}+\cos \left(\theta_{t-s}+\alpha\right) A_{2}=\Omega_{s}^{2}, \\
\dot{R}_{s}^{2}=-I_{2}\left(R_{t}\right)^{-1} \dot{R}_{t-s} I_{2}=-I_{2}\left(R_{t}\right)^{-1} R_{t-s} \Omega_{t-s} I_{2} \\
=\left(I_{2}\left(R_{t}\right)^{-1} R_{t-s} I_{2}\right)\left(-I_{2} \Omega_{t-s} I_{2}\right)=R_{s}^{2} \Omega_{s}^{2} .
\end{gathered}
$$

Действие отражения $\varepsilon^{2}$ на эластики представляется следующим образом:

$$
\begin{aligned}
& \varepsilon^{2}:\left(\begin{array}{l}
x_{s} \\
y_{s}
\end{array}\right) \stackrel{(1)}{\longmapsto}\left(\begin{array}{l}
x_{t-s} \\
y_{t-s}
\end{array}\right) \stackrel{(2)}{\longmapsto}\left(\begin{array}{l}
x_{t} \\
y_{t}
\end{array}\right)+\left(\begin{array}{cc}
-\cos 2 \chi & -\sin 2 \chi \\
-\sin 2 \chi & \cos 2 \chi
\end{array}\right)\left(\begin{array}{l}
x_{t-s} \\
y_{t-s}
\end{array}\right) \\
& \stackrel{(3)}{\longmapsto}\left(\begin{array}{cc}
-\cos 2 \chi & -\sin 2 \chi \\
\sin 2 \chi & -\cos 2 \chi
\end{array}\right)\left[\left(\begin{array}{l}
x_{t} \\
y_{t}
\end{array}\right)+\left(\begin{array}{cc}
-\cos 2 \chi & -\sin 2 \chi \\
-\sin 2 \chi & \cos 2 \chi
\end{array}\right)\left(\begin{array}{l}
x_{t-s} \\
y_{t-s}
\end{array}\right)\right] \\
&=\left(\begin{array}{l}
x_{t-s}-x_{t} \\
y_{t}-y_{t-s}
\end{array}\right)=\left(\begin{array}{l}
x_{s}^{2} \\
y_{s}^{2}
\end{array}\right),
\end{aligned}
$$

где $\chi$ - полярный угол точки $\left(x_{t}, y_{t}\right)$ :

$$
\cos \chi=\frac{x_{t}}{\sqrt{x_{t}^{2}+y_{t}^{2}}}, \quad \sin \chi=\frac{y_{t}}{\sqrt{x_{t}^{2}+y_{t}^{2}}} .
$$

То есть отражение $\varepsilon^{2}$ действует на эластики как композиция трех преобразований: (1) - обращение времени на эластике, $(2)$ - отражение плоскости $(x, y)$ в серединном перпендикуляре $l^{\perp}$ к ее хорде и $(3)-$ поворот на угол $(\pi-2 \chi)$.

Отражение $\varepsilon^{3}$. Отражение траекторий маятника $\left(\theta_{s}, c_{s}\right)$ в начале координат $(\theta, c)=(0,0)$ продолжается до следующей симметрии экстремальных траекторий:

$$
\begin{gathered}
\theta_{s}^{3}=-\theta_{s}, \quad c_{s}^{3}=-c_{s}, \quad \alpha^{3}=-\alpha, \\
x_{s}^{3}=x_{s}, \quad y_{s}^{3}=-y_{s}, \\
R_{s}^{3}=I_{2} R_{s} I_{2}, \quad \Omega_{s}^{3}=I_{2} \Omega_{s} I_{2} .
\end{gathered}
$$

ПреДЛОжЕНИЕ 4. Если $\left\{\lambda_{s} \mid s \in[0, t]\right\}$ - траектория системы (3.1)-(3.7), то кривая $\left\{\lambda_{s}^{3} \mid s \in[0, t]\right\}$ есть также траектория этой системы.

ДокАзАтЕльство. Аналогично доказательству предложения 3 проверим уравнение для матрицы вращения $R$ :

$$
\begin{gathered}
\Omega_{s}^{3}=\sin \left(\theta_{s}^{3}+\alpha^{3}\right) A_{1}-\cos \left(\theta_{s}^{3}+\alpha^{3}\right) A_{2}=-\sin \left(\theta_{s}+\alpha\right) A_{1}-\cos \left(\theta_{s}+\alpha\right) A_{2}, \\
I_{2} \Omega_{s} I_{2}=I_{2}\left[\sin \left(\theta_{s}+\alpha\right) A_{1}-\cos \left(\theta_{s}+\alpha\right) A_{2}\right] I_{2} \\
=-\sin \left(\theta_{s}+\alpha\right) A_{1}-\cos \left(\theta_{s}+\alpha\right) A_{2}=\Omega_{s}^{3}, \\
\dot{R}_{s}^{3}=I_{2} \dot{R}_{s} I_{2}=I_{2} R_{s} \Omega_{s} I_{2}=\left(I_{2} R_{s} I_{2}\right)\left(I_{2} \Omega_{s} I_{2}\right)=R_{s}^{3} \Omega_{s}^{3} .
\end{gathered}
$$


В отличие от отражений $\varepsilon^{1}$ и $\varepsilon^{2}$ отражение $\varepsilon^{3}$ не только переводит дуги экстремальных траекторий в дуги экстремальных траекторий, но и является симметрией гамильтоновой системы $\dot{\lambda}=\vec{H}(\lambda)$. Формулы (3.21)-(3.23) задают отображение $\varepsilon^{3}: T^{*} M \rightarrow T^{*} M$ такое, что для любой траектории $\lambda_{s}$ гамильтоновой системы ее образ $\varepsilon^{3}\left(\lambda_{s}\right)$ также является траекторией этой системы. Причем в случае отражения $\varepsilon^{3}$ сужение на отрезок времени $s \in[0, t]$ не требуется, так как отображение $\varepsilon^{3}(3.21)-(3.23)$ не зависит от конечного момента времени в отличие от отображений $\varepsilon^{1}(3.15)-(3.17)$ и $\varepsilon^{2}(3.18)-(3.20)$.

Отражение $\varepsilon^{3}$ имеет следующий геометрический смысл для эластик $\left(x_{s}, y_{s}\right)$ :

$$
\begin{aligned}
& \varepsilon^{3}:\left(\begin{array}{l}
x_{s} \\
y_{s}
\end{array}\right) \stackrel{(1)}{\longmapsto}\left(\begin{array}{cc}
\cos 2 \chi & \sin 2 \chi \\
\sin 2 \chi & -\cos 2 \chi
\end{array}\right)\left(\begin{array}{l}
x_{s} \\
y_{s}
\end{array}\right) \\
& \stackrel{(2)}{\longmapsto}\left(\begin{array}{cc}
\cos 2 \chi & \sin 2 \chi \\
-\sin 2 \chi & \cos 2 \chi
\end{array}\right)\left[\left(\begin{array}{cc}
\cos 2 \chi & \sin 2 \chi \\
\sin 2 \chi & -\cos 2 \chi
\end{array}\right)\left(\begin{array}{c}
x_{s} \\
y_{s}
\end{array}\right)\right]=\left(\begin{array}{c}
x_{s} \\
-y_{s}
\end{array}\right)=\left(\begin{array}{l}
x_{s}^{3} \\
y_{s}^{3}
\end{array}\right) \text {, }
\end{aligned}
$$

т.е. это композиция $(1)$ - отражения плоскости $(x, y)$ в хорде эластики, и $(2)-$ поворота на угол $(-2 \chi)$. С другой стороны,

$$
\varepsilon^{3}:\left(\begin{array}{l}
x_{s} \\
y_{s}
\end{array}\right) \mapsto\left(\begin{array}{c}
x_{s} \\
-y_{s}
\end{array}\right)=\left(\begin{array}{l}
x_{s}^{3} \\
y_{s}^{3}
\end{array}\right)
$$

есть отражение эластики в оси $x$.

3.2. Симметрии экспоненциального отображения. В этом пункте действие вращений $\Phi^{\beta}$ и отражений $\varepsilon^{i}$ в прообразе и образе экспоненциального отображения определяется так, чтобы они коммутировали с действием экспоненциального отображения.

Вращения $\Phi^{\beta}: \lambda \mapsto \lambda^{\beta}(3.9)-(3.14)$ являются симметриями гамильтоновой системы, поэтому их действие в $T^{*} M$ естественно распадается в прямую сумму действий в $N=T_{Q_{0}}^{*} M \times \mathbb{R}_{+}$(на $(\lambda, t)$, где $\lambda$ - начало экстремали) и в $M$ (на $Q_{t}$ - конце соответствующей экстремальной траектории):

$$
\begin{gathered}
\Phi^{\beta}: N \rightarrow N, \quad(\lambda, t) \mapsto\left(\lambda^{\beta}, t\right), \\
\lambda=(\theta, c, \alpha, r), \quad \lambda^{\beta}=\left(\theta, c, \alpha^{\beta}, r\right), \quad \alpha^{\beta}=\alpha+\beta,
\end{gathered}
$$

и

$$
\begin{gathered}
\Phi^{\beta}: M \rightarrow M, \quad Q \mapsto Q^{\beta} \\
Q=(x, y, R), \quad Q^{\beta}=\left(x^{\beta}, y^{\beta}, R^{\beta}\right), \\
\left(\begin{array}{l}
x^{\beta} \\
y^{\beta}
\end{array}\right)=\left(\begin{array}{cc}
\cos \beta & -\sin \beta \\
\sin \beta & \cos \beta
\end{array}\right)\left(\begin{array}{l}
x \\
y,
\end{array}\right), \quad R^{\beta}=e^{\beta A_{3}} R e^{-\beta A_{3}} .
\end{gathered}
$$

Действие отражений $\varepsilon^{i}$ в $N$ определяется ограничением их действия на вертикальные составляющие экстремальных траекторий в начальный момент времени $s=0$ :

$$
\begin{gathered}
\varepsilon^{i}: N \rightarrow N, \quad(\lambda, t) \mapsto\left(\lambda^{i}, t\right), \quad i=1,2,3, \\
\lambda=(\theta, c, \alpha, r), \quad \lambda^{i}=\left(\theta^{i}, c^{i}, \alpha^{i}, r\right),
\end{gathered}
$$


где $\lambda=\left.\lambda_{s}\right|_{s=0}, \lambda^{i}=\left.\lambda_{s}^{i}\right|_{s=0}$. Учитывая явные выражения действия отражений на дуги экстремальных траекторий из п. 3.1, получаем явные выражения для действия $\varepsilon^{i}$ в $N$

$$
\begin{aligned}
& \varepsilon^{1}:(\theta, c, \alpha, r, t) \mapsto\left(\theta^{1}, c^{1}, \alpha^{1}, r, t\right)=\left(\theta_{t},-c_{t}, \alpha+\pi, r, t\right), \\
& \varepsilon^{2}:(\theta, c, \alpha, r, t) \mapsto\left(\theta^{2}, c^{2}, \alpha^{2}, r, t\right)=\left(-\theta_{t}, c_{t}, \pi-\alpha, r, t\right), \\
& \varepsilon^{3}:(\theta, c, \alpha, r, t) \mapsto\left(\theta^{3}, c^{3}, \alpha^{3}, r, t\right)=(-\theta,-c,-\alpha, r, t) .
\end{aligned}
$$

Действие отражений в $M$ определяется их действием на экстремальные траектории в конечный момент времени $s=t$ :

$$
\begin{gathered}
\varepsilon^{i}: M \rightarrow M, \quad Q \mapsto Q^{i}, \quad i=1,2,3, \\
Q=(x, y, R), \quad Q^{i}=\left(x^{i}, y^{i}, R^{i}\right),
\end{gathered}
$$

где $Q=\left.Q_{s}\right|_{s=t}, Q^{i}=\left.Q_{s}^{i}\right|_{s=t}$. Явные формулы (по результатам п. 3.1) имеют вид

$$
\begin{aligned}
& \varepsilon^{1}:(x, y, R) \mapsto\left(x^{1}, y^{1}, R^{1}\right)=\left(-x,-y,(R)^{-1}\right), \\
& \varepsilon^{2}:(x, y, R) \mapsto\left(x^{2}, y^{2}, R^{2}\right)=\left(-x, y, I_{2}(R)^{-1} I_{2}\right), \\
& \varepsilon^{3}:(x, y, R) \mapsto\left(x^{3}, y^{3}, R^{3}\right)=\left(x,-y, I_{2} R I_{2}\right) .
\end{aligned}
$$

Итак, определено действие вращений и отражений в прообразе и образе экспоненциального отображения:

$$
\begin{aligned}
& \Phi^{\beta}, \varepsilon^{i}: N \rightarrow N, \quad(\lambda, t) \mapsto\left(\lambda^{\beta}, t\right),\left(\lambda^{i}, t\right), \\
& \Phi^{\beta}, \varepsilon^{i}: M \rightarrow M, \quad Q \mapsto Q^{\beta}, Q^{i} .
\end{aligned}
$$

Существенно, что образ $Q^{i}=\varepsilon^{i}(Q)$ зависит лишь от прообраза $Q$, но не от момента времени $t$.

ПРЕДлОЖЕНИЕ 5. Отображения $\Phi^{\beta}, \varepsilon^{i}$ являются симметриями экспоненицального отображения.

ДокАЗАТЕЛЬСтво следует из того, что $\Phi^{\beta}$, $\varepsilon^{i}$ преобразуют дуги экстремальных траекторий в дуги экстремальных траекторий, и из определения действия $\Phi^{\beta}, \varepsilon^{i}$ в $N, M$.

Рассмотрим группу симметрий экспоненциального отображения, порожденную вращениями и отражениями:

$$
G=\left\langle\Phi^{\beta}, \varepsilon^{1}, \varepsilon^{2}, \varepsilon^{3}\right\rangle .
$$

Таблица умножения в этой группе для действий $g: N \rightarrow N$ и $g: M \rightarrow M$, $g \in G$, совпадает с таблицей умножения для действия на траектории маятника $g:\left\{\left(\theta_{s}, c_{s}\right)\right\} \rightarrow\left\{\left(\theta_{s}^{i}, c_{s}^{i}\right)\right\}$ и имеет следующий вид:

\begin{tabular}{|c|c|c|c|c|}
\hline$\cdot \circ \cdot$ & $\varepsilon^{1}$ & $\varepsilon^{2}$ & $\varepsilon^{3}$ & $\Phi^{\beta}$ \\
\hline$\varepsilon^{1}$ & $\mathrm{Id}$ & $\varepsilon^{3}$ & $\varepsilon^{2}$ & $\Phi^{\beta} \circ \varepsilon^{1}$ \\
\hline$\varepsilon^{2}$ & $\varepsilon^{3}$ & $\mathrm{Id}$ & $\varepsilon^{1}$ & $\Phi^{-\beta} \circ \varepsilon^{2}$ \\
\hline$\varepsilon^{3}$ & $\varepsilon^{2}$ & $\varepsilon^{1}$ & $\mathrm{Id}$ & $\Phi^{-\beta} \circ \varepsilon^{3}$ \\
\hline$\Phi^{\gamma}$ & $\varepsilon^{1} \circ \Phi^{\gamma}$ & $\varepsilon^{2} \circ \Phi^{-\gamma}$ & $\varepsilon^{3} \circ \Phi^{-\gamma}$ & $\Phi^{\beta+\gamma}$ \\
\hline
\end{tabular}

Отсюда получаем явное описание группы симметрий

$$
G=\left\{\Phi^{\beta}, \Phi^{\beta} \circ \varepsilon^{i} \mid \beta \in S^{1}, i=1,2,3\right\} .
$$




\section{§4. Страты Максвелла, соответствующие симметриям}

Точка $Q_{t}$ экстремальной траектории $Q_{s}=\operatorname{Exp}(\lambda, s)$ называется точкой Максвелла, если существует другая экстремальная траектория $\widetilde{Q}_{s} \not \equiv Q_{s}$, для которой $\widetilde{Q}_{t}=Q_{t}$. Известно, что в аналитической субримановой задаче экстремальная траектория не может быть оптимальной после точки Максвелла (см., например, [18]).

Определим множество Максвелла в прообразе экспоненциального отображения:

$$
\operatorname{MAX}=\{(\lambda, t) \in N \mid \exists(\widetilde{\lambda}, t) \in N: \operatorname{Exp}(\lambda, s) \not \equiv \operatorname{Exp}(\widetilde{\lambda}, s), \operatorname{Exp}(\lambda, t)=\operatorname{Exp}(\widetilde{\lambda}, t)\} .
$$

Если $(\lambda, t) \in \mathrm{MAX}$, то экстремальная траектория $\operatorname{Exp}(\lambda, s)$ неоптимальна при $s>t$.

Определим также множество Максвелла, соответствующее группе $G$ :

$$
\begin{aligned}
\operatorname{MAX}^{G}=\{(\lambda, t) & \in N \mid \exists g \in G: \\
(\widetilde{\lambda}, t) & =g(\lambda, t), \operatorname{Exp}(\lambda, s) \not \equiv \operatorname{Exp}(\widetilde{\lambda}, s), \operatorname{Exp}(\lambda, t) \not \equiv \operatorname{Exp}(\widetilde{\lambda}, t)\},
\end{aligned}
$$

и множество Максвелла, соответствующее группе $\left\langle\varepsilon^{i}, \Phi^{\beta}\right\rangle, i=1,2,3$ :

$$
\begin{aligned}
\operatorname{MAX}^{i}=\{(\lambda, t) & \in N \mid \exists \beta \in S^{1}: \\
(\widetilde{\lambda}, t) & \left.=\varepsilon^{i} \circ \Phi^{\beta}(\lambda, t), \operatorname{Exp}(\lambda, s) \not \equiv \operatorname{Exp}(\widetilde{\lambda}, s), \operatorname{Exp}(\lambda, t)=\operatorname{Exp}(\widetilde{\lambda}, t)\right\} .
\end{aligned}
$$

Очевидно включение

$$
\operatorname{MAX}^{i} \subset \operatorname{MAX}^{G} \subset \operatorname{MAX}, \quad i=1,2,3 .
$$

Будем использовать следующие обозначения:

$$
\varepsilon^{i} \circ \Phi^{\beta}(\lambda, t)=\left(\lambda^{\beta, i}, t\right), \quad \operatorname{Exp}(\lambda, s)=Q_{s}, \quad \operatorname{Exp}\left(\lambda^{\beta, i}, s\right)=Q_{s}^{\beta, i} .
$$

Тогда

$$
\operatorname{MAX}^{i}=\left\{(\lambda, t) \in N \mid \exists \beta \in S^{1}: Q_{s} \not \equiv Q_{s}^{\beta, i}, Q_{t}=Q_{t}^{\beta, i}\right\} .
$$

Основная цель данного параграфа - вывод уравнений, задающих страты Максвелла $\mathrm{MAX}^{i}$. В п. 4.1 исследуются тождества $Q_{s} \equiv Q_{s}^{\beta, i}$, а в п. 4.2 - уравнение $Q_{t}=Q_{t}^{\beta, i}$. В заключительном п. 4.3 приведены окончательные результаты о стратах $\mathrm{MAX}^{i}$ и в качестве следствия - необходимые условия оптимальности в задаче о качении сферы по плоскости.

В следующем предложении показано, что разные начальные сопряженные векторы $\lambda, \widetilde{\lambda}$ могут задавать совпадающие экстремальные траектории $Q_{s} \equiv \widetilde{Q}_{s}$ только в случае, когда эластика $\left(x_{s}, y_{s}\right) \equiv\left(\widetilde{x}_{x}, \widetilde{y}_{s}\right)$ является прямой.

ПредЛОЖениЕ 6. Пусть $\lambda=\left(\theta, c, r, \alpha, Q_{0}\right), \widetilde{\lambda}=\left(\widetilde{\theta}, \widetilde{c}, \widetilde{r}, \widetilde{\alpha}, Q_{0}\right) \in C, \lambda_{s}=$ $e^{s \vec{H}}(\lambda)=\left(\theta_{s}, c_{s}, \alpha, r, Q_{s}\right), \widetilde{\lambda}_{s}=e^{s \vec{H}}(\widetilde{\lambda})=\left(\widetilde{\theta}_{s}, \widetilde{c}_{s}, \widetilde{\alpha}, \widetilde{r}, \widetilde{Q}_{s}\right)$. Тогда

$$
\begin{aligned}
& Q_{s} \equiv \widetilde{Q}_{s} \quad \Longleftrightarrow \quad \theta_{s}+\alpha \equiv \widetilde{\theta}_{s}+\widetilde{\alpha} \\
& \Longleftrightarrow \quad\left[\begin{array}{ll}
(1) & \lambda=\widetilde{\lambda} \\
(2) & c=\widetilde{c}=0, \quad \theta+\alpha=\widetilde{\theta}+\widetilde{\alpha}, \quad r \sin \theta=\widetilde{r} \sin \tilde{\theta}=0,
\end{array}\right.
\end{aligned}
$$

причем в случае $(2)$ эластика $\left(x_{s}, y_{s}\right) \equiv\left(\widetilde{x}_{x}, \widetilde{y}_{s}\right)$ есть прямая. 
ДокАЗАТЕЛЬСтво. Имеем цепочку эквивалентных тождеств:

$$
\begin{aligned}
Q_{s} \equiv \widetilde{Q}_{s} & \Longleftrightarrow x_{s} \equiv \widetilde{x}_{s}, \quad y_{s} \equiv \widetilde{y}_{s}, \quad R_{s} \equiv \widetilde{R}_{s} \quad \Longleftrightarrow \quad \theta_{s}+\alpha \equiv \widetilde{\theta}_{s}+\widetilde{\alpha} \\
& \Longleftrightarrow \theta_{s}+\alpha \equiv \widetilde{\theta}_{s}+\widetilde{\alpha}, \quad \widetilde{c}_{s} \equiv c_{s}, \quad r \sin \theta_{s} \equiv \widetilde{r}_{\sin } \widetilde{\theta}_{s} .
\end{aligned}
$$

А. Если $\widetilde{c}_{s} \equiv c_{s} \equiv 0$, то $\theta_{s}, \widetilde{\theta}_{s} \equiv$ const, и равенства (4.1) равносильны равенствам (2) в формулировке данного предложения.

Б. Пусть $\widetilde{c}_{s} \equiv c_{s} \not \equiv 0$. Дифференцируя третье тождество в (4.1), в силу гамильтоновой системы принципа максимума получаем $r \cos \theta_{s} \equiv \widetilde{r} \cos \widetilde{\theta}_{s}$, поэтому $r=\widetilde{r}$.

1) Если $r=\widetilde{r}=0$, то $\lambda_{s} \equiv \widetilde{\lambda}_{s}$ и $\lambda=\widetilde{\lambda}$.

2) Если $r=\widetilde{r} \neq 0$, то $\sin \theta_{s} \equiv \sin \widetilde{\theta}_{s}$, откуда получаем тождество $\sin \theta_{s}-$ $\sin \widetilde{\theta}_{s}=2 \cos \frac{\theta_{s}+\widetilde{\theta}_{s}}{2} \sin \frac{\widetilde{\alpha}-\alpha}{2} \equiv 0$.

2.1) Если $\widetilde{\alpha}-\alpha=0$, то $\lambda=\widetilde{\lambda}$.

$2.2)$ Если $\theta_{s}+\widetilde{\theta}_{s}=\pi+2 \pi k$, то $\theta_{s} \equiv \pi n, \widetilde{\theta}_{s} \equiv \pi m$, откуда следуют равенства (2) в формулировке данного предложения.

4.1. Неподвижные точки симметрий в прообразе экспоненциального отображения. В этом пункте получены необходимые условия выполнения тождества $Q_{s} \equiv Q_{s}^{\beta, i}$ в случае невырожденной эластики $\left(x_{s}, y_{s}\right)$. Как следует из предложения 6 , в этом случае тождество $Q_{s} \equiv Q_{s}^{\beta, i}$ эквивалентно равенству $\lambda=\lambda^{\beta, i}$.

ПреДЛОЖЕниЕ 7. Пусть $(\lambda, t) \in N,\left(\lambda^{\beta, i}, t\right)=\varepsilon^{i} \circ \Phi^{\beta}(\lambda, t), i \in\{1,2,3\}$. Пусть эластика $\gamma=\left\{\left(x_{s}, y_{s}\right) \mid s \in[0, t]\right\}$ невырождена. Если $Q_{s} \equiv Q_{s}^{\beta, i}$, то:

(i) в случае $i=1$ эластика $\gamma$ центрирована в вершине;

(ii) в случае $i=2$ эластика $\gamma$ центрирована в точке перегиба;

(iii) в случае $i=3$ тождество $Q_{s} \equiv Q_{s}^{\beta, i}$ невозможно.

Мы называем эластику $\gamma=\left\{\left(x_{s}, y_{s}\right) \mid s \in[0, t]\right\}$ центрированной в вершине (точке перегиба), если ее центр $\left(x_{t / 2}, y_{t / 2}\right)$ является вершиной, т.е. локальным экстремумом кривизны (соответственно точкой перегиба, т.е. точкой перемены знака кривизны).

ДокАЗАТЕльство. Если $Q_{s} \equiv Q_{s}^{\beta, i}$, то из предложения 6 следует равенство $\lambda=\lambda^{\beta, i}$. Поэтому для траекторий гамильтоновой системы принципа максимума $\lambda_{s}=\left(\theta_{s}, c_{s}, \alpha, r, Q_{s}\right)$ и $\lambda_{s}^{\beta, i}=\left(\theta_{s}^{\beta, i}, c_{s}^{\beta, i}, \alpha^{\beta, i}, r, Q_{s}^{\beta, i}\right)$ получаем $c_{s} \equiv c_{s}^{\beta, i}$.

(i) Если $i=1$, то $c_{s} \equiv c_{s}^{\beta, 1} \equiv c_{s}^{1} \equiv-c_{t-s}$. Кривизна эластики $c_{s}$ нечетна относительно точки $s=t / 2$, поэтому $c_{t / 2}=0$. Для невырожденной эластики любой нуль кривизны регулярен $\left(c_{s}=0 \Longrightarrow \dot{c}_{s} \neq 0\right)$, поэтому центр $\left(x_{t / 2}, y_{t / 2}\right)$ эластики $\gamma$ есть точка перегиба.

(ii) Если $i=2$, то $c_{s} \equiv c_{s}^{\beta, 2} \equiv c_{s}^{2} \equiv c_{t-s}$. Кривизна эластики $c_{s}$ четна относительно точки $s=t / 2$. Поэтому кривизна имеет локальный экстремум в точке $s=t / 2$, а эластика $\gamma$ - вершину в своем центре $\left(x_{t / 2}, y_{t / 2}\right)$.

(iii) Если $i=3$, то $\theta_{s} \equiv \theta_{s}^{3, \beta} \equiv-\theta_{s}$, т.е. $\theta_{s} \equiv 0$. Эластика $\gamma$ вырождается в прямую, что противоречит условию данного предложения. 
4.2. Неподвижные точки симметрий в образе экспоненциального отображения. В этом пункте исследуются точки $Q_{t}$ экстремальных траекторий, которые удовлетворяют уравнению $Q_{t}=Q_{t}^{\beta, i}$, возникающему в определении страта Максвелла $\mathrm{MAX}^{i}$. Получены уравнения многообразий в пространстве состояний $M$, содержащих эти точки. В этих уравнениях используется представление матрицы вращения $Q \in \mathrm{SO}(3)$ с помощью кватернионов $q=q_{0}+q_{1} i+q_{2} j+q_{3} k \in S^{3} \subset \mathbb{H}$ (см. $\left.\S 5\right)$. Соответствие $Q \mapsto q$ определяет кватернион $q$ с точностью до знака; это согласуется с тем, что возникающие далее уравнения для кватерниона инвариантны относительно инверсии $q \mapsto-q$ (см., например, (4.3)).

4.2.1. Уравнение $Q_{t}=Q_{t}^{\beta, 1}$. В предложении 8 описываются неподвижные точки действия группы симметрий $\left\langle\varepsilon^{1}, \Phi^{\beta}\right\rangle$ в образе экспоненциального отображения, т.е. такие точки $Q \in M$, что

$$
\exists \beta \in S^{1} \text { такое, что } \varepsilon^{1} \circ \Phi^{\beta}(Q)=Q .
$$

ПРеДЛОЖеНИЕ 8. Пусть $Q=(x, y, R) \in M u q=q_{0}+q_{1} i+q_{2} j+q_{3} k \in S^{3}-$ кватернион, соответствующий вращению $R \in \mathrm{SO}(3)$.

(i) $\operatorname{Eсли~}(x, y) \neq(0,0)$, то условие (4.2) равносильно дизгюнкиии

$$
q_{3}=0 \quad \text { илu } q= \pm k
$$

при этом $\beta=\pi$.

(ii) $\operatorname{Eсли~}(x, y)=(0,0)$, то из условия (4.3) следует условие (4.2), при этом можно положить $\beta=\pi$.

ДоказАтельство. Имеем

$$
\varepsilon^{1} \circ \Phi^{\beta}\left(\begin{array}{l}
x \\
y \\
R
\end{array}\right)=\left(\begin{array}{c}
-x \cos \beta+y \sin \beta \\
-x \sin \beta-y \cos \beta \\
e^{\beta A_{3}} R^{-1} e^{-\beta A_{3}}
\end{array}\right) .
$$

Далее,

$$
e^{\beta A_{3}} R^{-1} e^{-\beta A_{3}}=R \quad \Longleftrightarrow \quad R e^{\beta A_{3}} R e^{-\beta A_{3}}=\mathrm{Id} .
$$

(i) Пусть $(x, y) \neq(0,0)$. Тогда

$$
\left(\begin{array}{l}
-x \cos \beta+y \sin \beta \\
-x \sin \beta-y \cos \beta
\end{array}\right)=\left(\begin{array}{l}
x \\
y
\end{array}\right) \quad \Longleftrightarrow \quad \beta=\pi .
$$

Далее,

$$
\begin{gathered}
\varepsilon^{1} \circ \Phi^{\beta}(Q)=Q, \quad \beta=\pi \quad \Longleftrightarrow \quad R I_{3} R I_{3}=\mathrm{Id} \quad \Longleftrightarrow \quad\left(R I_{3}\right)^{2}=\mathrm{Id} \\
\Longleftrightarrow(q k)^{2}= \pm 1 \quad \Longleftrightarrow \quad q_{3}=0 \quad \text { или } q= \pm k .
\end{gathered}
$$

(ii) Если $(x, y)=(0,0)$, то, полагая $\beta=\pi$, так же, как в п. (i), заключаем, что из условия (4.3) следует условие (4.2). 
4.2.2. Уравнение $Q_{t}=Q_{t}^{\beta, 2}$. В предложении 9 описываются неподвижные точки действия группы симметрий $\left\langle\varepsilon^{2}, \Phi^{\beta}\right\rangle$ в образе экспоненциального отображения, т.е. такие точки $Q \in M$, что

$$
\exists \beta \in S^{1} \text { такое, что } \varepsilon^{2} \circ \Phi^{\beta}(Q)=Q .
$$

ПреДЛОЖеНИЕ 9. Пусть $Q=(x, y, R) \in M u q=q_{0}+q_{1} i+q_{2} j+q_{3} k \in S^{3}-$ кватернион, соответствующий вращению $R \in \mathrm{SO}(3)$.

(i) $\operatorname{Eсли~}(x, y)=(\rho \cos \chi, \rho \sin \chi) \neq(0,0)$, то условие (4.4) равносильно дизгюнкиии

$$
x q_{1}+y q_{2}=0 \quad \text { uлu } \quad q= \pm(\cos \chi i+\sin \chi j),
$$

при этом $\beta=\pi-2 \chi$.

(ii) Если $(x, y)=(0,0)$, то условие (4.4) выполняется. При этом $\beta$ можно определить из уравнения $q_{1} \sin (\beta / 2)+q_{2} \cos (\beta / 2)=0$.

ДокАзАтельство. Имеем

$$
\varepsilon^{2} \circ \Phi^{\beta}\left(\begin{array}{l}
x \\
y \\
R
\end{array}\right)=\left(\begin{array}{c}
-x \cos \beta+y \sin \beta \\
x \sin \beta+y \cos \beta \\
I_{2} e^{\beta A_{3}} R^{-1} e^{-\beta A_{3}} I_{2}
\end{array}\right)=\left(\begin{array}{c}
-x \cos \beta+y \sin \beta \\
x \sin \beta+y \cos \beta \\
e^{-\beta A_{3}} I_{2} R^{-1} e^{-\beta A_{3}} I_{2}
\end{array}\right) .
$$

Далее,

$$
\begin{aligned}
& e^{-\beta A_{3}} I_{2} R^{-1} e^{-\beta A_{3}} I_{2}=R \quad \Longleftrightarrow \quad R I_{2} e^{\beta A_{3}} R I_{2} e^{\beta A_{3}}=\mathrm{Id} \\
& \Longleftrightarrow\left(R I_{2} e^{\beta A_{3}}\right)^{2}=\mathrm{Id} \quad \Longleftrightarrow \quad q j\left(\cos \left(\frac{\beta}{2}\right)+\sin \left(\frac{\beta}{2}\right) k\right]^{2}= \pm 1 \\
& \Longleftrightarrow q\left[\sin \left(\frac{\beta}{2}\right) i+\cos \left(\frac{\beta}{2}\right) j\right]^{2}= \pm 1 \\
& \Longleftrightarrow \quad\left[\begin{array}{l}
\operatorname{Re}\left[q\left(\sin \left(\frac{\beta}{2}\right) i+\cos \left(\frac{\beta}{2}\right) j\right)\right]=0, \\
q\left(\sin \left(\frac{\beta}{2}\right) i+\cos \left(\frac{\beta}{2}\right) j\right)= \pm 1
\end{array}\right. \\
& \Longleftrightarrow \quad\left[\begin{array}{l}
q_{1} \sin \left(\frac{\beta}{2}\right)+q_{2} \cos \left(\frac{\beta}{2}\right)=0, \\
q= \pm\left(\sin \left(\frac{\beta}{2}\right) i+\cos \left(\frac{\beta}{2}\right) j\right) .
\end{array}\right.
\end{aligned}
$$

(i) Пусть $(x, y)=(\rho \cos \chi, \rho \sin \chi) \neq(0,0)$. Тогда

$$
\left(\begin{array}{c}
-\cos \beta x+\sin \beta y \\
\sin \beta x+\cos \beta y
\end{array}\right)=\left(\begin{array}{l}
x \\
y
\end{array}\right) \Longleftrightarrow \beta=\pi-2 \chi .
$$

Имеем

$$
\varepsilon^{2} \circ \Phi^{\beta}(Q)=Q, \quad \beta=\pi-2 \chi \Longleftrightarrow\left[\begin{array}{l}
q_{1} \cos \chi+q_{2} \sin \chi=0, \\
q= \pm(\cos \chi i+\sin \chi j),
\end{array}\right.
$$

что доказывает п. (i) данного предложения.

(ii) Если $(x, y)=(0,0)$, то равенство $\varepsilon^{2} \circ \Phi^{\beta}(Q)=Q$ равносильно дизъюнкции (4.5). Первому равенству $q_{1} \sin (\beta / 2)+q_{2} \cos (\beta / 2)=0$ можно удовлетворить, выбирая подходящее значение угла $\beta$. 
4.2.3. Уравнение $Q_{t}=Q_{t}^{\beta, 3}$. В предложении 10 описываются такие точки $Q \in M$, что

$$
\exists \beta \in S^{1} \text { такое, что } \varepsilon^{3} \circ \Phi^{\beta}(Q)=Q .
$$

ПреДЛОЖЕНИЕ 10. Пусть $Q=(x, y, R) \in M u q=q_{0}+q_{1} i+q_{2} j+q_{3} k \in S^{3}-$ кватернион, соответствующий вращению $R \in \mathrm{SO}(3)$.

(i) $\operatorname{Eсли~}(x, y) \neq(0,0)$, то условие (4.6) равносильно дизгюнкиии

$$
\left\{\begin{array} { l } 
{ x q _ { 1 } + y q _ { 2 } = 0 , } \\
{ q _ { 3 } = 0 }
\end{array} \text { или } \quad \left\{\begin{array}{l}
y q_{1}-x q_{2}=0, \\
q_{0}=0 .
\end{array}\right.\right.
$$

При этом $\beta=-2 \chi$.

(ii) $\operatorname{Eсли~}(x, y)=(0,0)$, то условие (4.6) равносильно дизгюнкиии

$$
q_{3}=0 \quad \text { или } \quad q_{0}=0 .
$$

При этом $\beta$ можно определить соответственно из уравнений

$$
\cos \left(\frac{\beta}{2}\right) q_{1}-\sin \left(\frac{\beta}{2}\right) q_{2}=0 \text { uлu } \sin \left(\frac{\beta}{2}\right) q_{1}+\cos \left(\frac{\beta}{2}\right) q_{2}=0 .
$$

ДоказАТЕЛьство. Имеем

$$
\varepsilon^{3} \circ \Phi^{\beta}(Q)=\left(\begin{array}{c}
x \cos \beta-y \sin \beta \\
-x \sin \beta-y \cos \beta \\
I_{2} e^{\beta A_{3}} R e^{-\beta A_{3}} I_{2}
\end{array}\right) .
$$

Далее, для любых $\beta \in S^{1}, R \in \mathrm{SO}(3)$ получаем цепочку эквивалентных равенств

$$
\begin{aligned}
I_{2} e^{\beta A_{3}} R e^{-\beta A_{3}} I_{2} \quad \Longleftrightarrow \quad I_{2} e^{\beta A_{3}} R=R I_{2} e^{\beta A_{3}} \\
\qquad \quad j\left(\cos \left(\frac{\beta}{2}\right)+\sin \left(\frac{\beta}{2}\right) k\right)\left(q_{0}+q_{1} i+q_{2} j+q_{3} k\right) \\
\quad= \pm\left(q_{0}+q_{1} i+q_{2} j+q_{3} k\right) j\left(\cos \left(\frac{\beta}{2}\right)+\sin \left(\frac{\beta}{2}\right) k\right) \\
\Longleftrightarrow \quad \begin{array}{l}
\cos \left(\frac{\beta}{2}\right) q_{3} i-\sin \left(\frac{\beta}{2}\right) q_{3} j+\left(\sin \left(\frac{\beta}{2}\right) q_{2}-\cos \left(\frac{\beta}{2}\right) q_{1}\right) k=0, \\
\sin \left(\frac{\beta}{2}\right) q_{1}+\cos \left(\frac{\beta}{2}\right) q_{2}-\sin \left(\frac{\beta}{2}\right) q_{0} i-\cos \left(\frac{\beta}{2}\right) q_{0} j=0
\end{array} \\
\Longleftrightarrow \quad\left\{\begin{array}{l}
\cos \left(\frac{\beta}{2}\right) q_{1}-\sin \left(\frac{\beta}{2}\right) q_{2}=0, \quad \text { или }\left\{\begin{array}{l}
\sin \left(\frac{\beta}{2}\right) q_{1}+\cos \left(\frac{\beta}{2}\right) q_{2}=0, \\
q_{0}=0 .
\end{array}\right.
\end{array}\right.
\end{aligned}
$$

(i) Пусть $(x, y)=(\rho \cos \chi, \rho \sin \chi) \neq(0,0)$, тогда

$$
\left\{\begin{array}{l}
x \cos \beta-y \sin \beta=x \\
-x \sin \beta-y \cos \beta=y
\end{array} \quad \Longleftrightarrow \beta=-2 \chi .\right.
$$


Поэтому $\varepsilon^{3} \circ \Phi^{\beta}(Q)=Q, \beta=-2 \chi$ равносильно дизъюнкции условий

$$
\left\{\begin{array} { l } 
{ q _ { 1 } \operatorname { c o s } \chi + q _ { 2 } \operatorname { s i n } \chi = 0 , } \\
{ q _ { 3 } = 0 }
\end{array} \text { или } \left\{\begin{array}{l}
q_{1} \sin \chi-q_{2} \cos \chi=0, \\
q_{0}=0,
\end{array}\right.\right.
$$

что при $(x, y) \neq(0,0)$ эквивалентно условиям (4.7).

(ii) Пусть $(x, y)=(0,0)$. Тогда угол $\beta$ в первых равенствах (4.9) можно подобрать, и условие (4.6) равносильно условиям (4.8).

4.3. Условия оптимальности экстремальных траекторий. В этом пункте получены основные результаты настоящей работы: условия принадлежности конечных точек экстремальных траекторий стратам Максвелла и, как следствие, необходимые условия оптимальности для экстремальных траекторий общего положения.

Tеорема 2. Пусть $t>0 u Q_{s}=\left(x_{s}, y_{s}, R_{s}\right)=\operatorname{Exp}(\lambda, s)$ есть такая экстремальная траектория, что:

(i) $q_{3}(t)=0$;

(ii) эластика $\left\{\left(x_{s}, y_{s}\right) \mid s \in[0, t]\right\}$ невырождена и не центрирована в точке перегиба.

Тогда $(\lambda, t) \in \mathrm{MAX}^{1}$, поэтому для любого $t_{1}>t$ траектория $Q_{s}, s \in\left[0, t_{1}\right]$, неоттимальна.

ДоКАЗАТЕЛЬСТво. Положим $\beta=\pi$. Тогда из предложения 8 следует, что $Q_{t}^{\beta, 1}=Q_{t}$, а из предложения 7 следует, что $Q_{s}^{\beta, 1} \not \equiv Q_{s}$. Поэтому $(\lambda, t) \in \operatorname{MAX}^{1}$.

Tеорема 3. Пусть $t>0 u Q_{s}=\left(x_{s}, y_{s}, R_{s}\right)=\operatorname{Exp}(\lambda, s)$ есть такая экстремальная траектория, что:

(i) $\left(x q_{1}+y q_{2}\right)(t)=0$;

(ii) эластика $\left\{\left(x_{s}, y_{s}\right) \mid s \in[0, t]\right\}$ невырождена и не центрирована в вершине.

Тогда $(\lambda, t) \in \mathrm{MAX}^{2}$, поэтому для любого $t_{1}>t$ траектория $Q_{s}, s \in\left[0, t_{1}\right]$, неоптимальна.

ДокАЗАТЕЛЬство. Выберем угол $\beta \in S^{1}$ следующим образом. Положим $\beta=\pi-2 \chi$, если $(x, y)(t)=(\rho \cos \chi, \rho \sin \chi) \neq(0,0)$. Если $(x, y)(t)=(0,0)$, то найдем $\beta$ из уравнения $q_{1}(t) \sin (\beta / 2)+q_{2}(t) \cos (\beta / 2)=0$.

Тогда из предложения 9 следует, что $Q_{t}^{\beta, 2}=Q_{t}$, а из предложения 7 следует, что $Q_{s}^{\beta, 2} \not \equiv Q_{s}$. Поэтому $(\lambda, t) \in \mathrm{MAX}^{2}$.

Теорема 4. Пусть $t>0 u Q_{s}=\left(x_{s}, y_{s}, R_{s}\right)=\operatorname{Exp}(\lambda, s)$ есть такая экстремальная траектория, что:

(i) $\left(x q_{1}+y q_{2}\right)(t)=q_{3}(t)=0$ или $\left(y q_{1}-x q_{2}\right)(t)=q_{0}(t)=0$;

(ii) эластика $\left\{\left(x_{s}, y_{s}\right) \mid s \in[0, t]\right\}$ невырождена.

Тогда $(\lambda, t) \in \mathrm{MAX}^{3}$, поэтому для любого $t_{1}>t$ траектория $Q_{s}, s \in\left[0, t_{1}\right]$, неоптимальна.

ДокАЗАТЕЛЬСтво. Из предложения 10 следует, что угол $\beta \in S^{1}$ можно подобрать так, что $Q_{t}^{\beta, 3}=Q_{t}$. Согласно п. (iii) предложения $7 Q_{s}^{\beta, 3} \not \equiv Q_{s}$. Поэтому $(\lambda, t) \in \mathrm{MAX}^{3}$. 
ЗАмечАниЕ. Учитывая то, что для любого кватерниона $q=q_{0}+i q_{1}+j q_{2}+$ $k q_{3} \in S^{3}$ соответствующее движение $R_{q}: \mathbb{R}^{3} \rightarrow \mathbb{R}^{3}$ есть вращение вокруг вектора $\left(q_{1}, q_{2}, q_{3}\right) \in \mathbb{R}^{3}$, можно дать следующую наглядную интерпретацию условий (i) теорем 2-4:

- условие (i) теоремы 2 означает, что вращение сферы $R_{t}$ есть поворот вокруг некоторой горизонтальной оси;

- условие (i) теоремы 3 означает, что вращение $R_{t}$ есть поворот вокруг некоторой оси, ортогональной вектору перемещения точки контакта сферы и плоскости $\left(x_{t}, y_{t}, 0\right)$;

- условие (i) теоремы 4 означает, что вращение $R_{t}$ есть поворот вокруг горизонтальной оси, отрогональной вектору $\left(x_{t}, y_{t}, 0\right)$, или что $R_{t}$ есть поворот на угол $\pi$ вокруг некоторой оси, лежащей в вертикальной плоскости, которая содержит вектор $\left(x_{t}, y_{t}, 0\right)$.

Для более полного исследования точек разреза желательно иметь эффективные оценки первых корней уравнений $q_{3}(t)=0$ и $\left(x q_{1}+y q_{2}\right)(t)=0$. Асимптотика этих оценок вблизи устойчивого положения равновесия маятника (т. е. при $(\theta, c) \rightarrow(0,0))$ была получена А. П. Маштаковым. Даже эта асимптотика имеет гораздо более сложную структуру, чем аналогичные глобальные оценки первого времени Максвелла в задаче Эйлера об эластиках (см. [12]), обобщенной задаче Дидоны (см. [18]) и субримановой задаче на группе движений плоскости (см. [19]). Поэтому задача об оптимальном качении сферы по плоскости гораздо сложнее этих родственных задач оптимального управления.

\section{§ 5. Приложение: представление вращений трехмерного пространства кватернионами}

Для представления ориентации катящейся сферы удобно наряду с матрицей $R \in \mathrm{SO}(3)$ использовать кватернионы. Напомним некоторые факты, связанные с этим представлением (см., например, [20], [21]). Рассмотрим тело кватернионов

$$
\mathbb{H}=\left\{q=q_{0}+i q_{1}+j q_{2}+k q_{3} \mid q_{0}, \ldots, q_{3} \in \mathbb{R}\right\},
$$

единичную сферу в нем

$$
S^{3}=\left\{\left.q \in \mathbb{H}|| q\right|^{2}=q_{0}^{2}+q_{1}^{2}+q_{2}^{2}+q_{3}^{2}=1\right\}
$$

и подпространство мнимых кватернионов

$$
I=\left\{q \in \mathbb{H} \mid \operatorname{Re} q=q_{0}=0\right\} \simeq \mathbb{R}^{3} .
$$

Любой кватернион $q \in S^{3}$ действует на евклидовом пространстве $I$ :

$$
q \in S^{3} \quad \Longrightarrow \quad R_{q}(a)=q a q^{-1}, \quad a \in I,
$$

причем отображение $R_{q}: I \rightarrow I$ является вращением, т.е. $R_{q} \in \mathrm{SO}_{3}(I)$. Отображение

$$
p: q \rightarrow R_{q}, \quad S^{3} \rightarrow \mathrm{SO}_{3}(I) \simeq \mathrm{SO}(3)
$$

является двулистным накрытием:

$$
R_{q}=R_{\widetilde{q}} \quad \Longleftrightarrow \quad q= \pm \widetilde{q} .
$$


Для любого кватерниона $q=q_{0}+i q_{1}+j q_{2}+k q_{3} \in S^{3}$ движение $R_{q}: I \rightarrow I$ есть вращение вокруг вектора $a=\operatorname{Im} q=i q_{1}+j q_{2}+k q_{3}$, так как

$$
R_{q}(a)=q a q^{-1}=\left(q_{0}+a\right) a\left(q_{0}+a\right)^{-1}=\left(q_{0}+a\right) a\left(q_{0}-a\right)=\left(q_{0}+a\right)\left(q_{0}-a\right) a=a,
$$

т.е. вектор $\operatorname{Im} q$ есть собственный вектор отображения $R_{q}$.

Любой кватернион $q \in S^{3}$ может быть записан в виде

$$
q=\cos \alpha+\sin \alpha u, \quad u \in S^{3} \cap I, \quad \alpha \in S^{1} .
$$

Тогда $R_{q}$ есть поворот пространства $I$ вокруг вектора $u$ на угол $2 \alpha$ : в правом ортонормированном базисе $(u, v, w)$ пространства $I$ этот поворот имеет матрицу

$$
R_{q}=\left(\begin{array}{ccc}
1 & 0 & 0 \\
0 & \cos (2 \alpha) & -\sin (2 \alpha) \\
0 & \sin (2 \alpha) & \cos (2 \alpha)
\end{array}\right)
$$

Поэтому

$$
\begin{gathered}
p\left(\cos \left(\frac{t}{2}\right)+\sin \left(\frac{t}{2}\right) i\right)=e^{t A_{1}}, \quad p\left(\cos \left(\frac{t}{2}\right)+\sin \left(\frac{t}{2}\right) j\right)=e^{t A_{2}}, \\
p\left(\cos \left(\frac{t}{2}\right)+\sin \left(\frac{t}{2}\right) k\right)=e^{t A_{3}}
\end{gathered}
$$

В работе было использовано следующее простое утверждение.

Лемма. Пусть $q \in S^{3} \subset \mathbb{H}$. Тогда:

(i) $q^{2}=1 \Longleftrightarrow q= \pm 1 \quad \Longleftrightarrow \quad \operatorname{Im} q=0$;

(ii) $q^{2}=-1 \quad \Longleftrightarrow \quad \operatorname{Re} q=0$.

Доказательство следует из равенства

$$
\left(q_{0}+q_{1} i+q_{2} j+q_{3} k\right)^{2}=q_{0}^{2}-q_{1}^{2}-q_{2}^{2}-q_{3}^{2}+2 q_{0}\left(q_{1} i+q_{2} j+q_{3} k\right) .
$$

\section{Список литературы}

[1] Robot motion planning and control, Lecture Notes in Control and Inform. Sci., 229, Springer-Verlag, Berlin-Heidelberg, 1998.

[2] Z. Li, J. Canny, "Motion of two rigid bodies with rolling constraint", IEEE Trans. on Robotics and Automation, 6:1 (1990), 62-72.

[3] A. Marigo, A. Bicchi, "Rolling bodies with regular surface: the holonomic case", Differential geometry and control (Boulder, CO, 1997), Proc. Sympos. Pure Math., 64, Amer. Math. Soc., Providence, RI, 1999, 241-256.

[4] А.А. Аграчев, Ю.Л. Сачков, Геометрическая теория управления, Физматлит, M., 2005.

[5] J. M. Hammersley, "Oxford commemoration ball", Probability, statistics and analysis, London Math. Soc. Lecture Note Ser., 79, Cambridge Univ. Press, Cambridge-New York, 1983, 112-142.

[6] A. M. Arthur, G. R. Walsh, "On Hammersley's minimum problem for a rolling sphere", Math. Proc. Cambridge Philos. Soc., 99:3 (1986), 529-534. 
[7] V. Jurdjevic, "The geometry of the plate-ball problem", Arch. Rational Mech. Anal., 124:4 (1993), 305-328.

[8] V. Jurdjevic, Geometric control theory, Cambridge Stud. Adv. Math., 52, Cambridge Univ. Press, Cambridge, 1997.

[9] Л. Эйлер, Метод нахождения кривых линий, обладающих свойствами максимума или минимума, или решение изопериметрической задачи, взятой в самом широком смысле, ГТТИ, М.-Л., 1934.

[10] А. Ляв, Математическая теория упругости, ОНТИ, М.-Л., 1935; пер. с англ.: A.E.H. Love, A treatise on the mathematical theory of elasticity, Cambridge Univ. Press, Cambridge, 1927.

[11] Ю. Л. Сачков, "Множество Максвелла в обобщенной задаче Дидоны", Maтем. сб., 197:4 (2006), 123-150; англ. пер.: Yu.L. Sachkov, "The Maxwell set in the generalized Dido problem", Sb. Math., 197:4 (2006), 595-621.

[12] Yu. L. Sachkov, "Maxwell strata in the Euler elastic problem", J. Dyn. Control Syst., 14:2 (2008), 169-234.

[13] Yu. L. Sachkov, "Conjugate and cut time in the sub-Riemannian problem on the group of motions of a plane", ESAIM Control Optim. Calc. Var., 16 (2009).

[14] O. Myasnichenko, "Nilpotent $(3,6)$ sub-Riemannian problem", J. Dynam. Control Systems, 8:4 (2002), 573-597.

[15] Ю. Л. Сачков, “Дискретные симметрии в обобщенной задаче Дидоны”, Матем. сб., 197:2 (2006), 95-116; англ. пер.: Yu. L. Sachkov, "Discrete symmetries in the generalized Dido problem", Sb. Math., 197:2 (2006), 235-257.

[16] U. Boscain, F. Rossi,, "Invariant Carnot-Caratheodory metrics on $S^{3}, \mathrm{SO}(3), \mathrm{SL}(2)$, and Lens spaces", SIAM J. Control Optim., 47:4 (2008), 1851-1878.

[17] Л. С. Понтрягин, В.Г. Болтянский, Р. В. Гамкрелидзе, Е. Ф. Мищенко, Математическая теория оптимальных прочессов, Наука, М., 1976; англ. пер.: L.S. Pontryagin, V. G. Boltyanskii, R. V. Gamkrelidze, E. F. Mishchenko, Selected works. Vol. 4. The mathematical theory of optimal processes, Classics Soviet Math., Gordon \& Breach, New York, 1986.

[18] Ю.Л. Сачков, "Полное описание стратов Максвелла в обобщенной задаче Дидоны", Матем. сб., 197:6 (2006), 111-160; англ. пер.: Yu. L. Sachkov, "Complete description of the Maxwell strata in the generalized Dido problem", Sb. Math., 197:6 (2006), 901-950.

[19] I. Moiseev, Yu. L. Sachkov, "Maxwell strata in sub-Riemannian problem on the group of motions of a plane", ESAIM Control Optim. Calc. Var., 16:2 (2010), 380-399.

[20] Л. С. Понтрягин, Обобщения чисел, Библиотека "Квант", 54, Наука, М., 1986; нем. пер.: L. S. Pontrjagin, Verallgemeinerungen der Zahlen, Akademie-Verlag, Berlin, 1991.

[21] Э.Т. Уиттекер, Аналитическая динамика, УРСС, М., 2004; пер. с англ.: E.T. Whittaker, A treatise on the analytical dynamics of particles and rigid bodies, Cambridge Math. Lib., Cambridge Univ. Press, Cambridge, 1988.

Ю. Л. Сачков (Yu. L. Sachkov)

Институт программных систем

им. А. К. Айламазяна РАН, г. Переславль-Залесский

E-mail: sachkov@sys.botik.ru
Поступила в редакцию 04.08.2009 и 03.03.2010 